VoL. 52 (1992) [137-151]

\title{
ON THE MAXIMAL CIRCUMRADIUS OF A PLANAR CONVEX SET CONTAINING ONE LATTICE POINT
}

\author{
Poh W. Awyong and Paul R. Scott
}

We obtain a result about the maximal circumradius of a planar compact convex set having circumcentre $O$ and containing no non-zero lattice points in its interior. In addition, we show that under certain conditions, the set with maximal circumradius is a triangle with an edge containing two lattice points.

\section{INTRODUCTION}

Let $K$ be a compact convex set in the plane and let $\Lambda$ be the integral lattice. We say that $K$ is $\Lambda$-admissible if the interior of $K$ contains the origin $O$, but no non-zero points of $\Lambda$. (This usage differs from the more conventional ' $\Lambda$ is $K$-admissible', but is more convenient here where $\Lambda$ is fixed and $K$ varies.)

A number of results concerning the circumradius of a general convex set are known (see for example $[1,2,4,5,6,8])$. However, there are relatively few results on the circumradius of a convex set constrained by lattice points (see for example [7]). In this paper we obtain a result on the circumradius of a planar, $\Lambda$-admissible, compact convex set having circumcentre $O$.

Let $S$ be the square with vertices $( \pm 1, \pm 1)$. We label the eight lattice points $(1,1),(0,1),(-1,1),(-1,0),(-1,-1),(0,-1),(1,-1),(1,0)$ on $S$ by $L_{1}, L_{2}, \ldots, L_{8}$ respectively. We also label the half edges, $L_{1} L_{2}, L_{2} L_{3}, \ldots, L_{8} L_{1}$ of $S, h_{1}, h_{2}, \ldots, h_{8}$ respectively. Let $O$ be the circumcentre of $K$ and let $C$ be the corresponding circumcircle. Suppose that $K$ crosses the half edge $h_{i}$. Then $h_{i}$ partitions $K$ into two regions, one of which does not contain $O$. Let $K^{\prime}$ denote this region. Then if $K^{\prime}$ intercepts $C$, we say that $K$ intercepts $C$ beyond the half edge $h_{i}$. We prove here the following result concerning the circumradius of $K$.

THEOREM. Let $K$ be a compact $\Lambda$-admissible convex set in the plane with circumcentre $O$, and circumradius $R(K)$. Then $R(K) \leqslant \alpha$ where $\alpha \approx 1.685$, unless $K$ intercepts $C$ beyond exactly two opposite half edges of $S$, in which case no upper bound can be found for $R(K)$. The bound $\alpha$ is best possible.

A set for which $R(K)=\alpha$ is attained is shown in Figure 1. The exact value for $\alpha$ is $\alpha=\sqrt{5 y^{2}+4 y+1}$ where $y$ is the positive root of $25 y^{3}+20 y^{2}-3=0$.

Received 12th October, 1994

Copyright Clearance Centre, Inc. Serial-fee code: 0004-9729/92 \$A2.00+0.00. 


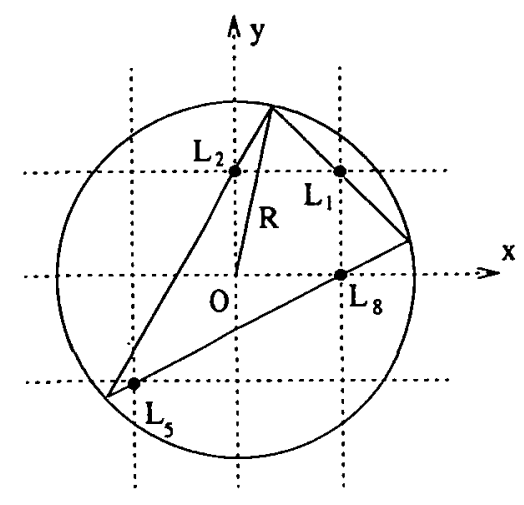

Figure 1: A set $K$ for which $R(K)=\alpha$.

\section{NARROWING THE SEARCH}

If $K$ lies within the square $S$, then $R(K) \leqslant \sqrt{2}<\alpha$. Hence we may assume that $R(K)>\sqrt{2}$, and we need only consider sets which extend beyond the boundary of $S$. Convexity arguments show that the convex set $K$ may cross at most four of the eight half edges of $S$, with no two of the crossed half edges comprising an edge of $S$. For a given set $K$, we call the set of half edges beyond which $K$ intercepts $C$ the intercept set of $K$.

It may be proved (see for example, $[9$, p. 59]) that either $C$ contains two points of the boundary of $K$ which are the ends of a diameter of $C$, or $C$ contains three points of the boundary of $K$ which form an acute-angled triangle containing $O$. Henceforth, we shall refer to this property of $C$ as the 'circumcircle property'. Clearly by the circumcircle property, $K$ must intercept $C$ beyond at least two half edges of $S$.

If $K$ intercepts $C$ beyond exactly two half edges of $S$, using rotations about $O$ and reflections in lines through $O$ to discard equivalent cases, we need only consider the cases where the intercept set of $K$ is
(a) $\left\{h_{1}, h_{3}\right\}$
(b) $\left\{h_{1}, h_{4}\right\}$
(c) $\left\{h_{1}, h_{5}\right\}$
(d) $\left\{h_{1}, h_{6}\right\}$
(e) $\left\{h_{1}, h_{8}\right\}$.

In cases (a) (b), (d) and (e), since $O$ is interior to $K$, all intercepts of $K$ and $C$ lie in the half planes $y>0, y>x, x>0$ and $y>0$ respectively. By the circumcircle property we can discard these cases.

In case (c) a $\Lambda$-admissible set $K$ can be constructed having circumcentre $O$ and diameter making a very small angle with the $y$-axis. It is easily seen that for such a set $K, R(K)$ may be made arbitrarily large. Therefore if $K$ intercepts $C$ beyond exactly two opposite half edges, no upper bound may be found for $R(K)$. 
We may now assume that $K$ intercepts $C$ beyond three or four half edges of $S$. By the circumcircle property, $C$ contains three points of the boundary of $K$ which form the vertices of an acute-angled triangle $T$ containing $O$. In the rest of the paper, we shall use 'triangle' to mean a $\Lambda$-admissible closed set bounded by the edges of a triangle with circumcentre $O$. As $R(T)=R(K)$, it is sufficient to establish the theorem for the class of triangles. Since $T$ crosses exactly three half edges of $S$, using rotations and reflections as before, we find that it suffices to consider the cases where the intercept set of $T$ is
(a) $\left\{h_{1}, h_{3}, h_{5}\right\}$
(b) $\left\{h_{1}, h_{3}, h_{8}\right\}$
(c) $\left\{h_{1}, h_{4}, h_{7}\right\}$
(d) $\left\{h_{1}, h_{4}, h_{8}\right\}$.

In case (b), since $O$ is interior to $K$, all intercepts of $K$ and $C$ lie in the half plane $y>0$. By the circumcircle property, this case may be eliminated.

We now let $F$ denote the family of triangles with circumcentre $O$ and having intercept set (a), (c) or (d). It will be shown in each of these cases that $F$ is contained in a closed disk. Therefore $R(T)$ is bounded and we let

$$
m=\sup _{T \in F} R(T)
$$

Let $\left\{T_{i}\right\}$ be a sequence in $F$ such that $R\left(T_{i}\right)$ converges to $m$. Since $T_{i}$ is contained in a closed disk, by Blaschke's selection theorem (see [1, p. 64]), there is a subsequence $\left\{T_{j}\right\}$ which converges to a triangle $\mathcal{T}$ in the Hausdorff metric. Since the circumradius function is continuous on $F$, we have

$$
R(\mathcal{T})=R\left(\lim _{j \rightarrow \infty} T_{j}\right)=\lim _{j \rightarrow \infty} R\left(T_{j}\right)=m
$$

Furthermore since $F$ is compact, $\mathcal{T} \in F$. For a given intercept set, a set $K$ with $R(K)=R(\mathcal{T})$ is referred to as a maximal set; in particular, a triangle $T$ with $R(T)=$ $R(\mathcal{T})$ is referred to as a maximal triangle.

In Section 3 we shall establish some properties of a maximal triangle. In Section 4, we prove two lemmas which will further narrow our search for a maximal set. In Section 5, Section 6 and Section 7, we shall prove the existence of a maximal triangle in cases (a), (c) and (d) respectively. For each case we shall also establish the uniqueness of the maximal triangle by employing the results in Section 4 to eliminate all other triangles in $F$ as possible solutions.

Unless otherwise specified, the vertices of a triangle will be described in an anticlockwise order. 


\section{Properties of a maximal triangle and a MAXimal Set}

The following lemmas establish some properties of a maximal triangle and a maximal set for intercept sets (a), (c) or (d) in Section 2.

LEMMA 1. If $T$ is a maximal triangle, then each edge of $T$ must contain at least one non-zero lattice point in its interior.

Proof: Let $T=\triangle X Y Z$ be a maximal triangle with edges $x, y$ and $z$ lying opposite the vertices $X, Y$ and $Z$ respectively. We suppose that there is at least one edge of $T$ which does not contain a non-zero lattice point in its interior. If edge $x$ (say) contains no non-zero lattice point in its interior, we enlarge $T$ about $X$ until $x$ first contains such a lattice point. Denote this enlarged triangle $T^{\prime}$ with vertices $X^{\prime}, Y^{\prime}, Z^{\prime}$ and edges $x^{\prime}, y^{\prime}, z^{\prime}$. Let $K^{\prime}$ be the set bounded by the circumcircle of $T$ and the edges $x^{\prime}, y^{\prime}$ and $z^{\prime}$. Clearly $K^{\prime}$ is $\Lambda$-admissible and $R\left(K^{\prime}\right)=R(T)$. By construction, $K^{\prime}$ is bounded by three straight edges and two arc boundaries of $C$. If now $y^{\prime}$ contains no non-zero lattice point in its interior, a small enlargement of $T^{\prime}$ about $Y^{\prime}$ results in a triangle $T^{\prime \prime}$ containing no non-zero lattice point in its interior. By construction, the set bounded by the edges of $T^{\prime \prime}$ and the circumcircle of $T$ has three arc boundaries. On the other hand, if $y^{\prime}$ contains a lattice point in its interior, a small clockwise rotation about the lattice point (choose the lattice point closest to $X^{\prime}$ if there is more than one lattice point in the interior of $y^{\prime}$ ) also results in a set with three arc boundaries. We may therefore assume that $K^{\prime}$ is a set with three arc boundaries. But now a small enlargement of $C$ about $O$ to $C^{\prime}$ will result in a $\Lambda$ admissible set $K^{\prime \prime}$ bounded by the lines containing the straight edges of $K^{\prime}$ and arcs of $C^{\prime}$. Clearly $R\left(K^{\prime \prime}\right)>R\left(K^{\prime}\right)=R(T)$, contradicting our assumption that $T$ is a maximal triangle.

LEMMA 2. If $K$ is a maximal set, then $K$ is a triangle.

Proof: We suppose that $K$ is not a triangle. Then by the circumcircle property, $K$ intercepts $C$ in three points which form an acute-angled triangle $T$ containing $O$. Since $K$ is a maximal set, $T$ is a maximal triangle. As $K \neq T$, there is an edge $e$ of $T$ whose interior lies in the interior of $K$. Hence $e$ contains no non-zero lattice point in its interior. By Lemma $1, T$ is not a maximal triangle. Hence $K$ is a triangle.

If each edge of a triangle contains exactly one non-zero lattice point in its interior, we call the join of a vertex to the opposite lattice point a $V L$-line.

LEMMA 3. If $T$ is a maximal triangle then either

(i) its $V L$-lines are concurrent or

(ii) at least one of the edges of $T$ contains two lattice points in its interior.

Proof: Let $T=\triangle X Y Z$ be a maximal triangle. By Lemma 1, each edge of $T$ 
contains at least one lattice point in its interior. Suppose there is exactly one lattice point, $L_{x}, L_{y}$ and $L_{z}$ lying in the interior of each of the edges $x, y$ and $z$ respectively and suppose that the $V L$-lines, $X L_{x}, Y L_{y}$ and $Z L_{z}$ are not concurrent. We transform the edges of the triangle $T$ by first rotating the edge $X Y$ (sliding the endpoints on $C$ ) about $L_{z}$ through a small angle $\theta$ to $X^{\prime} Y^{\prime}$. This is followed by a rotation in the same direction of the edge $Y Z$ about $L_{x}$ to the edge $Y^{\prime} Z^{\prime}$. Finally $Z X$ is rotated in the same direction about $L_{y}$ to $Z^{\prime} X^{\prime \prime}$. We denote the described transformation on the edges of $T$ by $f_{c}(T, \theta)$ if the edges of $T$ are rotated in a clockwise manner, and by $f_{a}(T, \theta)$, if the edges of $T$ are rotated in an anticlockwise direction. We note that $\angle Y X X^{\prime}=\angle X^{\prime} Y^{\prime} Y$ since these two angles are in the same segment of $C$ subtended by chord $X^{\prime} Y$. Similarly, $\angle Y^{\prime} Y X=\angle Y^{\prime} X^{\prime} X$. We therefore deduce that $\triangle Y L_{z} Y^{\prime}$ and $\triangle X^{\prime} L_{z} X$ are similar. Hence

$$
\frac{X X^{\prime}}{Y Y^{\prime}}=\frac{X^{\prime} L_{z}}{L_{z} Y} .
$$

Since $X, Y$ and $Z$ are oriented anticlockwise and $\theta$ is small and $\triangle X Y Z$ contains the circumcentre in its interior, $f_{a}(T, \theta)$ gives $X^{\prime} L_{z}=X L_{z}-\varepsilon$, where $\varepsilon$ is small and positive. Therefore

$$
\frac{X X^{\prime}}{Y Y^{\prime}}=\frac{X L_{z}-\varepsilon}{L_{z} Y}<\frac{X L_{z}}{L_{z} Y} .
$$

Similarly

$$
\frac{Y Y^{\prime}}{Z Z^{\prime}}<\frac{Y L_{x}}{L_{x} Z}
$$

and

$$
\frac{Z Z^{\prime}}{X X^{\prime \prime}}<\frac{Z L_{y}}{L_{y} X}
$$

Multiplying, we obtain

$$
\frac{X X^{\prime}}{X X^{\prime \prime}}<\frac{X L_{z}}{L_{z} Y} \cdot \frac{Y L_{x}}{L_{x} Z} \cdot \frac{Z L_{y}}{L_{y} X}=p, \quad \text { say }
$$

where $X X^{\prime} / X X^{\prime \prime}$ differs from $p$ by a small amount $\varepsilon_{p}$. Similarly, if $f_{c}(T, \theta)$ is applied,

$$
\frac{X X^{\prime}}{X X^{\prime \prime}}>p
$$

where $X X^{\prime} / X X^{\prime \prime}$ differs from $p$ by a small amount $\varepsilon_{p}^{\prime}$. We note that by Ceva's theorem (see [3, p. 90]), $p=1$ if and only if the $V L$-lines $X L_{x}, Y L_{y}$ and $Z L_{z}$ are concurrent. By assumption, $p \neq 1$.

If $p>1, f_{a}(T, \theta)$ yields $X X^{\prime} / X X^{\prime \prime}=p-\varepsilon_{p}$. We choose $\theta$ so that $\varepsilon_{p}$ is sufficiently small to give $X X^{\prime} / X X^{\prime \prime}>1$. Since now $X X^{\prime}>X X^{\prime \prime}$, the transformation results in a 


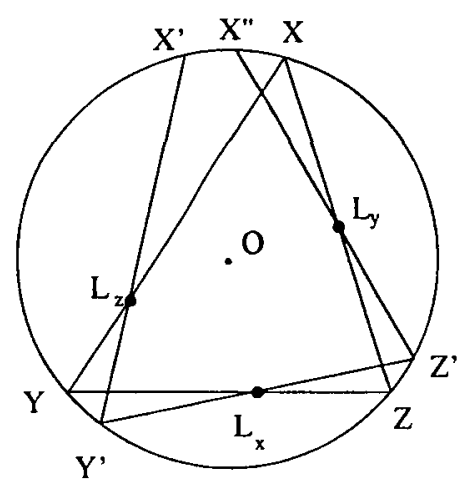

Figure 2: The effect of $f_{\alpha}(T, \theta)$ on $T$ for $p>1$.

set $K$ bounded by edges $X^{\prime} Y^{\prime}, Y^{\prime} Z^{\prime}, Z^{\prime} X^{\prime \prime}$ and the $\operatorname{arc} X^{\prime \prime} X^{\prime}$ as shown in Figure 2. By Lemma 2, $K$ is not a maximal set. Since $R(K)=R(T), T$ is therefore not a maximal triangle, contradicting our assumption.

If $p<1, f_{c}(T, \theta)$ yields $X X^{\prime} / X X^{\prime \prime}=p+\varepsilon_{p}^{\prime}$. We choose $\theta$ so that $\varepsilon_{p}^{\prime}$ is sufficiently small to give $X X^{\prime} / X X^{\prime \prime}<1$. Since now $X X^{\prime}<X X^{\prime \prime}$, the transformation results in a set $K$ with an arc boundary. Arguing as before, $T$ is not a maximal triangle.

Therefore, the maximal triangle is such that either its $V L$-lines are concurrent, or it has at least one edge containing two lattice points in its interior.

For future easy reference, we summarise our findings thus far in the following lemma.

LEMMA 4. A maximal set $K$ is a triangle having a non-zero lattice point interior to each of its edges and such that either

(i) its VL-lines are concurrent or

(ii) at least one of its edges contains two lattice points in its interior.

Proof: By Lemima 2, a maximal set $K$ is a triangle. By Lemma 1, a maximal triangle has a non-zero lattice point interior to each of its edges and by Lemma 3, a maximal triangle has concurrent $V L$-lines or has at least one edge containing two lattice points.

\section{NARROWING THE SEARCH FURTHER}

Henceforth we may restrict the members of $F$ to those triangles with a given intercept set and with edges each containing a non-zero lattice point. For each intercept set we list the possible lattice points contained in each of the three edges of a triangle in $F$. 


\begin{tabular}{cccc} 
Intercept set & Edge 1 & Edge 2 & Edge 3 \\
\hline$\left\{h_{1}, h_{3}, h_{5}\right\}$ & $L_{2}$ & $L_{4}$ & $L_{6}$ or $L_{1}$ or both \\
$\left\{h_{1}, h_{4}, h_{7}\right\}$ & $L_{8}$ & $L_{2}$ or $L_{4}$ or both & $L_{5}$ or $L_{7}$ or both \\
$\left\{h_{1}, h_{4}, h_{8}\right\}$ & $L_{1}$ & $L_{2}$ or $L_{4}$ or both & $L_{5}$ or $L_{8}$ or both
\end{tabular}

To each $T=\triangle X Y Z$, we associate the ordered set $\left(\ell_{1}, \ell_{2}, \ell_{3}\right)$ called the latticepoint set where $\ell_{i}, i=1,2,3$ is a listing of the non-zero lattice points in the interior of the edges $Y Z, Z X$ and $X Y$ respectively. If $T$ has an edge $X Y$ say, containing in its interior two non-zero lattice points $L_{z}$ and $L_{z}^{\prime}$ in the order $X, L_{z}, L_{z}^{\prime}, Y$, then we write $\ell_{3}=L_{z} L_{z}^{\prime}$.

We shall now prove two lemmas to help us narrow down the possibilities for a maximal triangle. Lemma 5 establishes the uniqueness of a triangle $T^{*}=\Delta X^{*} Y^{*} Z^{*}$ with a given lattice-point set $\left(L_{x}, L_{y}, L_{z} L_{z}^{\prime}\right)$. Therefore from the above table, it may be deduced that there are at most five such triangles satisfying condition (ii) of Lemma 4 (see also Figure 3).

Lemma 5. Let $T=\triangle X Y Z$ and $T^{\prime}=\Delta X^{\prime} Y^{\prime} Z^{\prime}$ be two triangles with the same intercept set and lattice-point set $\left(L_{x}, L_{y}, L_{z} L_{z}^{\prime}\right)$. Then $T=T^{\prime}$.

Proof: We first suppose that $R\left(T^{\prime}\right)>R(T)$. Then $X^{\prime}$ lies on the side of $X$ remote from $Y$, and $Y^{\prime}$ lies on the side of $Y$ remote from $X$. The edge $X^{\prime} Z^{\prime}$ is therefore oriented clockwise about $L_{y}$ from $X Z$ and the edge $Y^{\prime} Z^{\prime}$ is oriented anticlockwise about $L_{x}$ from $Y Z$. The vertex $Z^{\prime}$ therefore lies in the interior of $T$ and so $O$ is not the circumcentre of $T^{\prime}$, contradicting our assumption on $T^{\prime}$. Hence $R\left(T^{\prime}\right) \ngtr R(T)$. A similar argument shows that $R\left(T^{\prime}\right) \nless R(T)$. Therefore $R\left(T^{\prime}\right)=R(T)$. It follows that $X^{\prime}=X, Y^{\prime}=Y$ so $Z^{\prime}=Z$ and therefore $T^{\prime}=T$.

The next lemma helps us to eliminate those triangles in $F$ which do not satisfy conditions (i) and (ii) of Lemma 4 . We shall be comparing a triangle $T^{*}=\triangle X^{*} Y^{*} Z^{*}$ along with its given lattice-point set $\left(L_{x}, L_{y}, L_{z} L_{z}^{\prime}\right)$ with a related triangle $T=\triangle X Y Z$ having the same intercept set as $T$.

Lemma 6. Let $T^{*}=\triangle X^{*} Y^{*} Z^{*}$ denote the unique triangle with a given latticepoint set $\left(L_{z}, L_{y}, L_{z} L_{z}^{\prime}\right)$. We define $P^{*}$ to be the intersection of $X^{*} L_{x}$ and $Y^{*} L_{y}$, and $Q^{*}$ to be the intersection of $Z^{*} P^{*}$ produced with $X^{*} Y^{*}$. If $L_{z}$ lies in the open line segment $Q^{*} X^{*}$, then any triangle with lattice-point set $\left(L_{z}, L_{y}, L_{z}\right)$ is not maximal. If $L_{z}^{\prime}$ lies in the open line segment $Q^{*} Y^{*}$, then any triangle with lattice-point set $\left(L_{z}, L_{y}, L_{z}^{\prime}\right)$ is not maximal.

Proof: Let $T=\triangle X Y Z$ be a triangle with the lattice-point set $\left(L_{x}, L_{y}, L_{z}\right)$. We define $P$ to be the intersection of $X L_{z}$ and $Y L_{y}$, and $Q$ to be the intersection of $Z P$ produced with $X Y$. We show that $Z L_{z}$ cannot pass through $P$. It will then follow that the $V L$-lines of $T$ are not concurrent and by Lemma 3, $T$ is not a maximal 


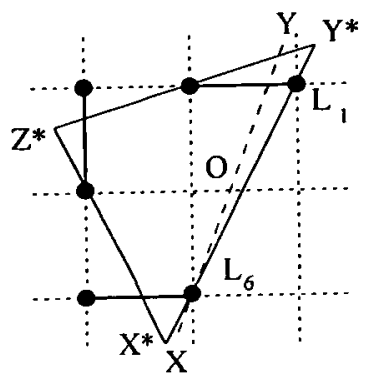

(a) $\left\{h_{1}, h_{3}, h_{5}\right\}$

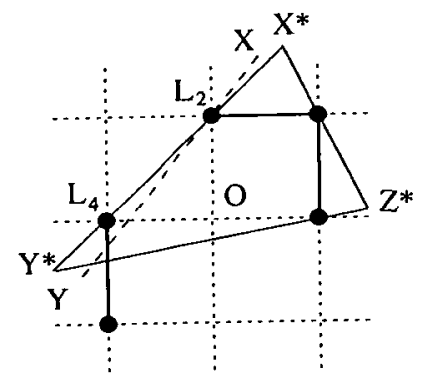

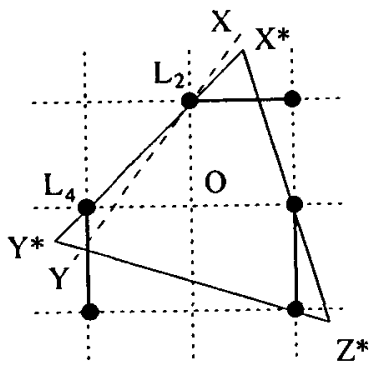

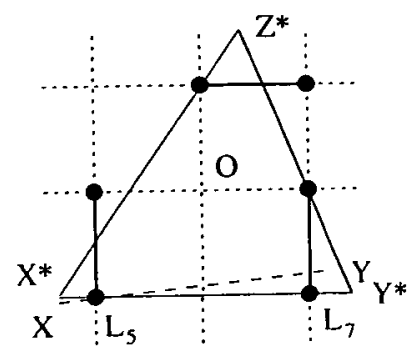

(b) $\left\{h_{1}, h_{4}, h_{7}\right\}$

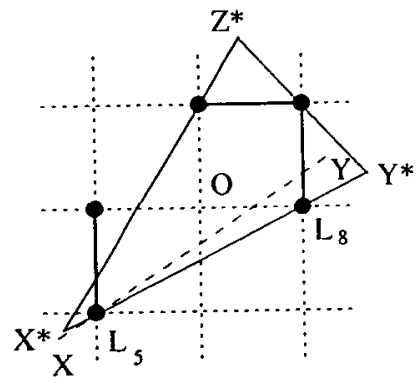

(c) $\left\{h_{1}, h_{4}, h_{8}\right\}$

Figure 3: Triangles with edges containing two lattice points.

triangle. Let $h_{x}$ and $h_{y}$ be the open half planes bounded by the line $Q^{*} Z^{*}$ containing $X^{*}$ and $Y^{*}$ respectively.

Figure 3 shows the five possible triangles $T^{*}$, and how the intercept set constrains the edge $X Y$ of any triangle $T=\triangle X Y Z$ with lattice point set $\left(L_{x}, L_{y}, L_{z}\right)$. Since $T$ is $\Lambda$-admissible, $T$ cannot contain $L_{z}^{\prime}$ in its interior. Thus in each case, since $X^{*}, L_{z}$, $L_{z}^{\prime}$ and $Y^{*}$ are in the given order, the edge $X Y$ of $T$ is oriented anticlockwise about $L_{z}$ from the edge $X^{*} Y^{*}$ of $T^{*}$. Similarly if $T$ has lattice-point set $\left(L_{x}, L_{y}, L_{z}^{\prime}\right)$, then it cannot contain $L_{z}$ in its interior and the edge $X Y$ of $T$ is oriented clockwise about $L_{z}^{\prime}$ from $X^{*} Y^{*}$.

We first suppose that $T$ is a maximal triangle. Therefore $R(T) \geqslant R\left(T^{*}\right)$ which implies that the vertices of $T$ are exterior to $T^{*}$. As observed from Figure 3, the edge $X Y$ of $T$ is oriented anticlockwise about $L_{z}$ from the edge $X^{*} Y^{*}$ of $T^{*}$, and since the vertices of $T$ are exterior to $T^{*}$, the edges $Y Z$ and $Z X$ of $T$ are also oriented anticlockwise about $L_{x}$ and $L_{y}$ respectively from the corresponding edges of $T^{*}$ (see 
Figure 4).

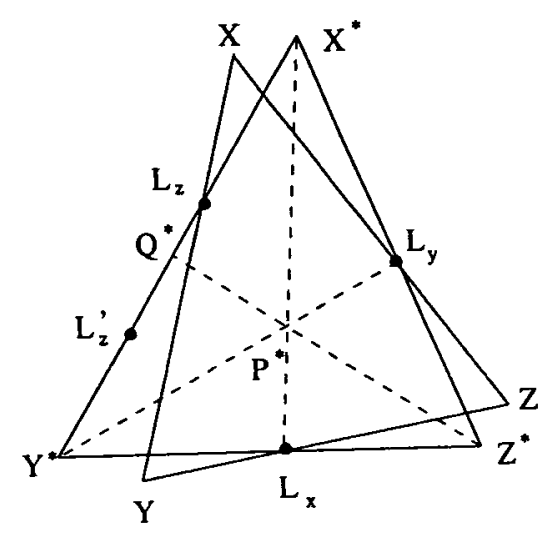

Figure 4: The case where $R(T) \geqslant R\left(T^{*}\right)$.

Therefore the $V L$-lines, $X L_{x}$ and $Y L_{y}$ of $T$ are oriented anticlockwise about $L_{x}$ and $L_{y}$ from $X^{*} L_{x}$ and $Y^{*} L_{y}$ respectively, placing the point $P$ in the interior of $\triangle Y^{*} P^{*} L_{z}$. It follows that $\mathrm{P}$ lies in $h_{y}$. Since $L_{z}$ lies in the open line segment $Q^{*} X^{*}$, the lattice point $L_{z}$ lies in $h_{x}$. Also, since the edges $Y Z$ and $Z X$ are oriented anticlockwise about $L_{x}$ and $L_{y}$ respectively from $Y^{*} Z^{*}$ and $Z^{*} X^{*}$, the point $Z$ necessarily lies in $h_{x}$. Hence the closed line segment $Z L_{z}$ is contained in $h_{x}$. Therefore the closed line segment $Z L_{z}$ and the point $P$ are on opposite sides of the line $Q^{*} Z^{*}$ and hence the $V L$-lines of $T$ are not concurrent. Therefore $T$ is not a maximal triangle.

We now let $T=\triangle X Y Z$ be a triangle with the lattice-point set $\left(L_{x}, L_{y}, L_{z}^{\prime}\right)$. Arguing in a similar way as above, the edge $X Y$ of $T$ is oriented clockwise about $L_{z}^{\prime}$ from $X^{*} Y^{*}$. Defining $P$ and $Q$ as above and using a similar argument, it may be shown that the closed line segment $Z L_{z}$ and the point $P$ again lie on opposite sides of the line $Q^{*} Z^{*}$. Hence the $V L$-lines of $T$ are not concurrent and $T$ is not a maximal triangle.

In the subsequent sections, we shall employ Lemma 6 to show that a maximal triangle in $F$ has an edge containing two lattice points in its interior. The maximal triangle may then be found by evaluating the circumradius for each triangle with an edge containing two interior lattice points.

We now list all lattice-point sets for a given intercept set. We shall employ the notation used in Lemma 6 throughout the rest of the paper. In addition, we shall denote by $V_{i}$, the vertex of $T$ lying beyond $h_{i}$. 


\section{THE $\left\{h_{1}, h_{3}, h_{5}\right\}$ CASE}

Let $F$ denote the family of triangles with circumcentre $O$ and intercept set $\left\{h_{1}, h_{3}, h_{5}\right\}$. The following lattice-point sets occur for $F$ :
(a) $\left(L_{2}, L_{4}, L_{6} L_{1}\right)$
(b) $\left(L_{2}, L_{4}, L_{6}\right)$
(c) $\left(L_{2}, L_{4}, L_{1}\right)$.

Let $T$ be in $F$. Due to the constraints of the given intercept set, the edge of $T$ containing $L_{2}$ has a slope between 0 and 1 , and the edge containing $L_{4}$ has a slope less than -1 . It follows that the vertex $V_{3}$ may not be further from the origin than the point $(-2,1)$. Hence $F$ is contained in the closed disk centred at $O$, radius $\sqrt{5}$ and by Blaschke's selection theorem, a maximal triangle $\mathcal{T}$ may be found in $F$.

In case (a) we let $T^{*}=\Delta V_{5} V_{1} V_{3} \equiv \Delta X^{*} Y^{*} Z^{*}$ be the unique triangle with latticepoint set $\left(L_{2}, L_{4}, L_{6} L_{1}\right)$. We assign the coordinates $(x, 2 x-1)$ and $(y, 2 y-1)$ to $X^{*}$ and $Y^{*}$ respectively. Since $X^{*}$ and $Y^{*}$ also lie on $C$,

$$
x^{2}+(2 x-1)^{2}=y^{2}+(2 y-1)^{2}
$$

which gives $x+y=4 / 5$. We let $Z^{*}$ have coordinates $\left(z_{1}, z_{2}\right)$. Since $Y^{*} Z^{*}$ and $Z^{*} X^{*}$ contain the lattice points $(0,1)$ and $(-1,0)$ respectively,

$$
\frac{z_{2}-1}{z_{1}-0}=\frac{2 y-2}{y}, \quad \frac{z_{2}-0}{z_{1}+1}=\frac{2 x-1}{x+1} .
$$

Eliminating $x$ and solving for $z_{1}$ and $z_{2}$, we obtain

$$
z_{1}=\frac{-y(6+5 y)}{25 y-18}, \quad z_{2}=-\frac{-23 y+10 y^{2}+6}{25 y-18} .
$$

As $Z^{*}$ also lies on the circumcircle,

$$
z_{1}^{2}+z_{2}^{2}=y^{2}+(2 y-1)^{2}
$$

Simplifying and factorising, we have

$$
(5 y-2)\left(25 y^{3}-45 y^{2}+25 y-6\right)=0 \text {. }
$$

Since $y>1$, we solve $25 y^{3}-45 y^{2}+25 y-6=0$ to obtain $y \approx 1.080$ and $R\left(T^{*}\right) \approx$ $1.582<\alpha$.

We now proceed to show that any triangle $T$ with lattice-point set (b) or (c) is not maximal. We consider the quadrangle $X^{*} Y^{*} L_{2} L_{4}$. Let $K$ be the point of intersection of the lines $X^{*} Y^{*}$ and $L_{2} L_{4}$, that is the point $(2,3)$. By the harmonic property of the quadrangle, the points $X^{*}, Y^{*}$ separate $Q^{*}$ and $K$ harmonically. Therefore the 
cross-ratio $\left(X^{*}, Y^{*} ; Q^{*}, K\right)=-1$. We now orthogonally project the four points $X^{*}$, $Y^{*}, Q^{*}$ and $K$ on the $x$-axis to obtain the points $X_{x}^{*}, Y_{x}^{*}, Q_{x}^{*}$ and $K_{x}$ respectively. Since the cross-ratio is unaltered under projection, we have $\left(X_{x}^{*}, Y_{x}^{*} ; Q_{x}^{*}, K_{x}\right)=-1$. Letting the $x$-coordinate of $Q^{*}$ be $q$, we have

$$
\frac{q-x}{y-q}=-\frac{2-x}{y-2}
$$

which gives $q \approx 0.689$. Therefore $L_{6}$ lies in the open line segment $Q^{*} X^{*}$ and $L_{1}$ lies in the open line segment $Q^{*} Y^{*}$. By Lemma 6, any triangle with lattice-point set (b) or $(c)$ is not maximal.

Hence $\mathcal{T}$ is the triangle with lattice-point set $\left(L_{2}, L_{4}, L_{6} L_{1}\right)$ and $R(\mathcal{T}) \approx 1.582<$ $\alpha$.

\section{THE $\left\{h_{1}, h_{4}, h_{7}\right\}$ CASE}

Let $F$ denote the family of triangles with circumcentre $O$ and intercept set $\left\{h_{1}, h_{4}, h_{7}\right\}$. The following lattice-point sets occur for $F$ :
(a) $\left(L_{8}, L_{4}, L_{5} L_{7}\right)$
(b) $\left(L_{5}, L_{8}, L_{2} L_{4}\right)$
(c) $\left(L_{8}, L_{2} L_{4}, L_{5} L_{7}\right)$
(d) $\left(L_{7}, L_{8}, L_{2} L_{4}\right)$
(e) $\left(L_{8}, L_{2}, L_{5} L_{7}\right)$
(f) $\left(L_{4}, L_{5}, L_{8}\right)$
(g) $\left(L_{7}, L_{8}, L_{4}\right)$
(h) $\left(L_{8}, L_{2}, L_{5}\right)$
(i) $\left(L_{8}, L_{2}, L_{7}\right)$.

Let $T$ be in $F$. As before, given the intercept set of $T$, the edge of $T$ containing $L_{2}$ has a slope which lies between $1 / 2$ and 1 , and the edge of $T$ containing $L_{8}$ has a slope greater than 1 . It follows that the vertex $V_{1}$ may not be further from the origin than the point $(1,3)$. Hence $F$ is contained in the closed disk centred at $O$, radius $\sqrt{10}$ and by Blaschke's selection theorem, a maximal triangle $\mathcal{T}$ may be found in $F$.

We first consider a triangle $T$ with lattice-point set (a), (b), (c) or (f). We note that in these cases, $\left|O V_{1}\right|<\sqrt{5}$ and $\left|O V_{4}\right| \geqslant \sqrt{5}$. Hence $T$ may not have circumcentre $O$. We may therefore eliminate cases (a), (b), (c) and (f). (In fact, any triangle with lattice-point set $(f)$ contains the lattice point $(-2,-1)$ in its interior and is therefore not $\Lambda$-admissible.)

We now let $T^{*}=\triangle V_{1} V_{4} V_{7} \equiv \triangle X^{*} Y^{*} Z^{*}$ be the triangle with lattice-point set (d). We assign the coordinates $(x, x+1)$ to $X^{*}, 0<x<1$. Since $X^{*}$ and $Y^{*}$ lie on $C$, by symmetry $Y^{*}$ has coordinates $(-x-1,-x)$. We let $Z^{*}$ have coordinates $\left(z_{1}, z_{2}\right)$. Since $Z^{*} X^{*}$ and $Y^{*} Z^{*}$ contain the lattice points $(1,0)$ and $(1,-1)$ respectively,

$$
\frac{z_{2}-0}{z_{1}-1}=\frac{x+1}{x-1}, \quad \frac{z_{2}+1}{z_{1}-1}=\frac{-x+1}{-x-2}
$$


Solving for $z_{1}$ and $z_{2}$, we obtain

As $Z^{*}$ also lies on $C$,

$$
z_{1}=-\frac{x^{2}-4 x-3}{5 x+1}, \quad z_{2}=-\frac{x^{2}+3 x+2}{5 x+1} .
$$

$$
z_{1}^{2}+z_{2}^{2}=x^{2}+(x+1)^{2} .
$$

Simplifying and factorising, we obtain

$$
(2 x+1)\left(2 x^{3}+2 x^{2}-1\right)=0 .
$$

Since $0<x<1$, we solve $2 x^{3}+2 x^{2}-1=0$ to obtain $x \approx 0.565$ and $R\left(T^{*}\right) \approx 1.664<$ $\alpha$.

We now show that any triangle $T$ with lattice-point set $(\mathrm{g})$ is not maximal. We consider the quadrangle formed by the points $X^{*} Y^{*} L_{8} L_{7}$. Letting the $x$-coordinate of $Q^{*}$ be $q$ and considering cross-ratios as in Section 4 , we find that $q \approx 0.256$. Therefore $L_{4}$ lies in the open line segment $Q^{*} Y^{*}$. By Lemma 6, any triangle with lattice-point set $(\mathrm{g})$ is not maximal.

We now let $T^{*}=\triangle V_{4} V_{7} V_{1} \equiv \triangle X^{*} Y^{*} Z^{*}$ be the triangle with lattice-point set (e). We assign the coordinates $(1+y,-1)$ to $Y^{*}, 0<y<1$. Since $X^{*} Y^{*}$ also lie on $C$, by symmetry, $X^{*}$ has coordinates $(-1-y,-1)$. We let $Z^{*}$ have coordinates $\left(z_{1}, z_{2}\right)$. Since $Y^{*} Z^{*}$ and $Z^{*} X^{*}$ contain the lattice points $(1,0)$ and $(0,1)$ respectively,

$$
\frac{z_{2}-0}{z_{1}-1}=\frac{1}{-y}, \quad \frac{z_{2}-1}{z_{1}-0}=\frac{2}{1+y} .
$$

Solving for $z_{1}$ and $z_{2}$, we obtain

$$
z_{1}=\frac{-\left(y^{2}-1\right)}{1+3 y}, \quad z_{2}=\frac{3+y}{1+3 y} .
$$

As $Z^{*}$ also lies on $C$,

$$
z_{1}^{2}+z_{2}^{2}=(1+y)^{2}+1 .
$$

Simplifying and factorising, we obtain

$$
(1+y)\left(y^{3}+2 y^{2}+2 y-1\right)=0 .
$$

Since $0<y<1$, we solve $\left(y^{3}+2 y^{2}+2 y-1\right)=0$ for $y$ to obtain $y \approx 0.353$ and $R\left(T^{*}\right) \approx 1.683<\alpha$.

We now show that any triangle $T$ with lattice-point set (h) or (i) is not maximal. We consider the quadrangle $X^{*} Y^{*} L_{8} L_{2}$. Letting the $x$-coordinate of $Q^{*}$ be $q$ and using cross-ratios as in Section 5 , we find that $q \approx 0.915$. Therefore $L_{5}$ lies in the open line segment $Q^{*} X^{*}$ and $L_{7}$ lies in the open line segment $Q^{*} Y^{*}$. By Lemma 6 , any triangle with lattice-point set (h) or (i) is not maximal.

Hence $\mathcal{T}$ is the triangle with lattice-point set $\left(L_{8}, L_{2}, L_{5} L_{7}\right)$ and $R(\mathcal{T}) \approx 1.683<$ $\boldsymbol{\alpha}$. 


\section{THE $\left\{h_{1}, h_{4}, h_{8}\right\}$ CASE}

Let $F$ denote the family of triangles with circumcentre $O$ and intercept set $\left\{h_{1}, h_{4}, h_{8}\right\}$. The following lattice-point sets occur for $F$ :
(a) $\left(L_{1}, L_{4}, L_{5} L_{8}\right)$
(b) $\left(L_{5}, L_{1}, L_{2} L_{4}\right)$
(c) $\left(L_{1}, L_{2} L_{4}, L_{5} L_{8}\right)$
(d) $\left(L_{8}, L_{1}, L_{2} L_{4}\right)$
(e) $\left(L_{1}, L_{2}, L_{5} L_{8}\right)$
(f) $\left(L_{1}, L_{4}, L_{5}\right)$
(g) $\left(L_{8}, L_{1}, L_{4}\right)$
(h) $\left(L_{1}, L_{2}, L_{8}\right)$
(i) $\left(L_{1}, L_{2}, L_{5}\right)$.

Let $T$ be in $F$. As before, given the intercept set of $T$, the edge of $T$ containing $L_{2}$ has a slope between $1 / 2$ and 1 and the edge containing $L_{1}$ has a slope less than 0 . It follows that the vertex $V_{1}$ may not be further from the origin than the point $(1,3)$. Hence $F$ is contained in the closed disk centred at $O$, radius $\sqrt{10}$ and by Blaschke's selection theorem, a maximal triangle $\mathcal{T}$ may be found in $F$.

We first consider a triangle with lattice-point set (a), (b), (c) or (f). We note that in all these cases, $\left|O V_{1}\right|<\sqrt{5}$ and $\left|O V_{4}\right| \geqslant \sqrt{5}$. Hence $T$ may not have circumcentre $O$. Therefore we may disregard cases (a), (b), (c) and (f). (In fact, any triangle with lattice-point set $(a)$ or (f) contains the lattice-point $(-2,-1)$ in its interior and is therefore not $\Lambda$-admissible.)

We now let $T^{*}=\triangle V_{1} V_{4} V_{8} \equiv \triangle X^{*} Y^{*} Z^{*}$ be the triangle with lattice-point set (d). We assign the coordinates $(x, x+1)$ to $X^{*}$. Since $X^{*}$ and $Y^{*}$ also lie on $C$, by symmetry, $Y^{*}$ has coordinates $(-x-1,-x)$. We let $Z^{*}$ have coordinates $\left(z_{1}, z_{2}\right)$. Since $Z^{*} X^{*}$ and $Y^{*} Z^{*}$ contain the lattice points $(1,1)$ and $(1,0)$ respectively,

$$
\frac{z_{2}-1}{z_{1}-1}=\frac{x}{x-1}, \quad \frac{z_{2}-0}{z_{1}-1}=\frac{-x}{-x-2} \text {. }
$$

Solving for $z_{1}$ and $z_{2}$, we obtain

$$
z_{1}=\frac{-x^{2}+2 x+2}{3 x}, \quad z_{2}=\frac{-x+1}{3} .
$$

As $Z^{*}$ also lies on $C$,

$$
z_{1}^{2}+z_{2}^{2}=x^{2}+(x+1)^{2} .
$$

Simplifying and factorising, we obtain

$$
(2 x+1)\left(2 x^{3}+2 x^{2}-1\right)=0 .
$$

Since $0<x<1$, we solve $2 x^{3}+2 x^{2}-1=0$ for $x$ to obtain $x \approx 0.565$ and $R\left(T^{*}\right) \approx$ $1.664<\alpha$. 
We now proceed to show that any triangle $T$ with lattice-point set (g) is not maximal. We consider the quadrangle $X^{*} Y^{*} L_{8} L_{1}$. Using the cross-ratio argument, we find that the $x$-coordinate $q$ of $Q^{*}$ is approximately 0.256 and therefore $L_{4}$ lies in the open line segment $Q^{*} Y^{*}$. By Lemma 6, any triangle with lattice-point set (g) is not maximal.

We now let $T^{*}=\triangle V_{4} V_{8} V_{1} \equiv \triangle X^{*} Y^{*} Z^{*}$ be the triangle with lattice-point set (e). We assign the coordinates $(2 x+1, x)$ and $(2 y+1, y)$ to $X^{*}$ and $Y^{*}$ respectively. Since $X^{*}$ and $Y^{*}$ also lie on $C$,

$$
(2 x+1)^{2}+x^{2}=(2 y+1)^{2}+y^{2}
$$

which gives $x+y=-\frac{4}{5}$. We let $Z^{*}$ have coordinates $\left(z_{1}, z_{2}\right)$. Since $Y^{*} Z^{*}$ and $Z^{*} X^{*}$ contain the lattice points $(1,1)$ and $(0,1)$ respectively,

$$
\frac{z_{2}-1}{z_{1}-1}=\frac{y-1}{2 y}, \quad \frac{z_{2}-1}{z_{1}-0}=\frac{x-1}{2 x+1}
$$

Eliminating $x$ and solving for $z_{1}$ and $z_{2}$ in terms of $y$, we obtain

$$
z_{1}=-\frac{-3-7 y+10 y^{2}}{25 y+3}, \quad z_{2}=-\frac{-12-21 y+5 y^{2}}{25 y+3}
$$

As $Z^{*}$ also lies on $C$,

$$
z_{1}^{2}+z_{2}^{2}=(2 y+1)^{2}+y^{2} .
$$

Simplifying and factorising we obtain

$$
(5 y+2)\left(25 y^{3}+20 y^{2}-3\right)=0
$$

Since $y>0$, we solve $25 y^{3}+20 y^{2}-3=0$ for $y$ to obtain $y \approx 0.326$ and $R\left(T^{*}\right) \approx$ $1.685=\alpha$.

We now show that any triangle $T$ with lattice-point set (h) or (i) is not maximal. We consider the quadrangle $X^{*} Y^{*} L_{1} L_{2}$ and using cross-ratios as before, we show that the $x$-coordinate $q$ of $Q^{*}$ is approximately 0.953 . Therefore $L_{5}$ lies in the open line segment $Q^{*} X^{*}$ and $L_{8}$ lies in the open line segment $Q^{*} Y^{*}$. By Lemma 6 , any triangle with lattice-point set (h) or (i) is not maximal.

Hence $\mathcal{T}$ is the triangle with lattice-point set $\left(L_{1}, L_{2}, L_{5} L_{8}\right)$ and $R(\mathcal{T}) \approx 1.685=$ $\alpha$. We note that $\alpha=\sqrt{(2 y+1)^{2}+y^{2}}$, where $y \approx 0.326$.

Comparing the results in Section 5, Section 6 and Section 7. we conclude that the maximal set $K$ is the triangle with lattice-point set $\left(L_{1}, L_{2}, L_{5} L_{8}\right)$ with $R(K) \approx 1.685$. The theorem is therefore proved. 


\section{CoMment}

It is interesting to observe that the triangles with lattice-point sets $\left\{L_{7}, L_{8}, L_{2} L_{4}\right\}$ in Section 6 and $\left\{L_{8}, L_{1}, L_{2} L_{4}\right\}$ in Section 7 have the same circumradius. There does not appear to be any obvious algebraic connection, and we have been unable to find a simple geometrical proof.

\section{REFERENCES}

[1] H.G. Eggleston, Convexity (Cambridge University Press, 1958).

[2] H.G. Eggleston, 'Notes on Minkowski geometry (I): Relations between the circumradius, diameter, inradius and minimal width of a convex set', J. London Math. Soc. 33 (1958), 76-81.

[3] E.A. Maxwell, Geometry for advanced pupils (Oxford University Press, 1949).

[4] M. Henk and G.A. Tsintsifas, 'Some inequalities for planar convex figures', Elem. Math. 49 (1994), 120-124.

[5] P.R. Scott, 'Two inequalities for convex sets in the plane', Bull. Austral. Math. Soc. 19 (1978), 131-133.

[6] P.R. Scott, 'A family of inequalities for convex sets', Bull. Austral. Math. Soc. 20 (1979), 237-245.

[7] P.R. Scott, 'Further inequalities for convex sets with lattice point constraints in the the plane', Bull. Austral. Math. Soc. 21 (1980), 7-12.

[8] P.R. Scott, 'Sets of constant width and inequalities', Quart. J. Math. Oxford Ser. (2) 32 (1981), 345-348.

[9] I.M.Yaglom and V.G. Boltyanskii, Convex figures, Translated by P.J. Kelly and L.F. Walton (Holt, Rinehart and Winston, New York, 1961).

Department of Pure Mathematics

The University of Adelaide

South Australia 5005

e-mail: pawyong@maths.adelaide.edu.au

pscott@maths.adelaide.edu.au 